\title{
Configurable Executable Task Models Supporting the Transition from Design Time to Runtime
}

\author{
Birgit Bomsdorf, Stefan Grau, Martin Hudasch, and Jan-Torsten Milde* \\ Department of Applied Computer Science, University of Applied Sciences Fulda, \\ Marquardstr. 35, D-36039 Fulda, Germany \\ \{bomsdorf, milde\} @hs-fulda.de
}

\begin{abstract}
Model-based design of user interfaces mostly starts with task and domain modeling. The resulting models are an important input to subsequent development steps. Thus, a thorough evaluation of these specifications is of great importance, e.g. to avoid the implementation of bad or even error prone solutions. Executable task models are in use for several years to evaluate the design time specifications. They are also used at runtime by now as part of the final application. In this paper we propose an executable task model that is configured using the design time model. Kernel concept of this work is a task state machine describing a generic task life cycle assigned to each task. Developers may extend it at design time to describe application dependent behavior. The extensions are automatically transferred to the runtime system. A further focus of the paper is on the specification of temporal relations and their extensibility in terms of model description and execution.
\end{abstract}

Keywords: task modeling, executable task model, runtime support, temporal relations.

\section{Introduction}

In model-based design of user interfaces it is well established to distinguish different modeling layers [1]: Starting with a model of the tasks formulated from the users' perspective (task model) and a model of entities involved in task execution (domain model) a model of the abstract user interface (dialog and presentation model) is developed. This model is transformed into one or more concrete UI models taking into account context information such as availability of media, modes and devices. All in all, results of task modeling are an important input to the subsequent development steps. A task model, however, may be very complex. It describes not only the tasks users should perform to reach their goals by means of a system under consideration, but also their hierarchical decomposition into subtasks, information on task sequencing and conditions of task execution. . Executable task models are in use for several years to evaluate such specification [2], [3], [4], [5]. They are also used at runtime by now as a component of the final application [6], [7], [8], [9], [10].

Executable task models at runtime are based on generic task life cycles in the works mentioned above. They are represented by means of state machines showing a

\footnotetext{
* Corresponding authors.
} 
lot of similarities with respect to states and transitions. In our work, in contrast to the other approaches, the state machine is not only part of the runtime system but additionally used as an explicit modeling concept at design time [11]. Specifications by the developers extend the generic state machine of a task and are systematically adopted by the runtime system. Basically, similar to [8] and to [9], we follow the approach of model interpretation at runtime. The design time models are used to configure the executable task model. This work aims at supporting the transition from design time to runtime. Its main ideas are presented in the following. In section 2 basic modeling concepts including the generic state machine are introduced. In the subsequent section 3 the generic state machine as used at runtime and steps of the configuration process are presented. Remarks concerning the state of the work are given in section 4 .

\section{Modeling Concepts}

The kernel modeling concepts of the approach presented here are Task, Condition, Temporal Relation, Behavior and Domain Object that are linked to each other via different, predefined relations. The concepts are shortly introduced below except Domain Object, which is used to specify objects that are involved in task execution. The object model is not further described here since in our current work we focus on tasks and temporal relations. Basically an object-oriented approach is used that could be described by means of UML. Examples of linking objects with other concepts are given within the following introduction of the concepts Behavior and Condition.

\subsection{Task Specification}

Task structures, as in general, are defined by means of hierarchical decomposition of tasks (CompositeTask) into their subtasks. A task not subdivided into further tasks is called an elementary task (ElementaryTask). These bottom tasks of a task hierarchy are the "points" where the intended actions affecting the domain are performed. Composite tasks are high level tasks structuring a task space.

The time period within which a task is executed is used as an explicit concept to describe temporal dependencies. A task defines a frame (task frame) of its execution and thus for the actions performed to fulfill it. In the case of a composite task each subtask possesses a frame of its own while the superior task's frame includes all of them. Overall, temporal dependencies are based on time periods (as in the work by Allen [12]), e.g. constraints on task duration (temporal constraints), but also on temporal relations (as often used in task modeling, e.g. in MAD [5] and CTT [3]).

Temporal Constraints. Temporal constraints, defined as task attributes in the metamodel, specify delays before (StartOffset) and after (EndOffset) a task run. In addition, the duration of task execution can be constrained in terms of a minimum and a maximum period of time. For example, a user might move the icon of a text document into a workspace by means of a defined touch-based gesture. The user can repeat this several times but the system should take care of a clear separation of the 
different repetitions. Fig. 1 (a) shows a solution: The required behavior is modeled by a Loop of the interaction task move icon into workspace together with a value specification for its EndOffset defining a delay between two executions. MinDuration and MaxDuration might be set to specify the period of time within which the gesture is to be performed.

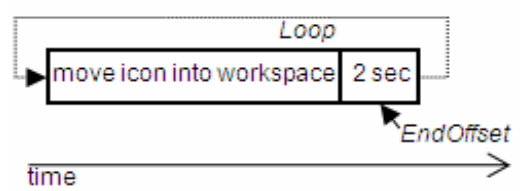

Fig. 1. Loop of a task with a time interval of 2 seconds after each performance

Generic Task Life Cycle at Design Time. Tasks undergo different state changes while being performed. These states are also significant to users since in their planning of follow-up activities they take into account current task situations. It is important to a user, for example, whether he can start to work on a task or not (because of unfulfilled conditions), or if he is already performing a task and thus its subtasks. The states and possible state changes are described by means of a generic state machine. It and the description of its states are shown in Fig. 2. The transitions between the task states are labeled with the events triggering them.

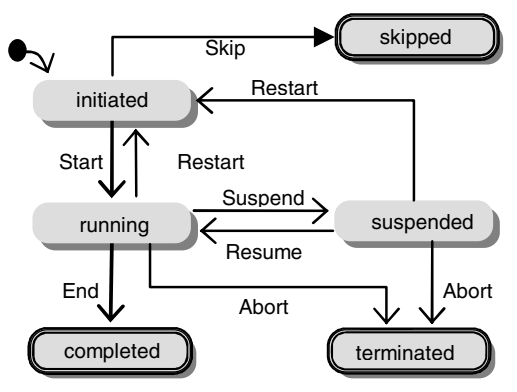

\begin{tabular}{|c|c|}
\hline State & Meaning \\
\hline initiated & $\begin{array}{l}\text { if all preconditions are fulfilled the task } \\
\text { can be started }\end{array}$ \\
\hline skipped & the task is omitted \\
\hline running & $\begin{array}{l}\text { denotes the actual performance of the } \\
\text { task and of its subtasks, if applicable }\end{array}$ \\
\hline completed & marks a successful task execution \\
\hline suspended & the task is interrupted \\
\hline terminated & indicates an abort \\
\hline
\end{tabular}

Fig. 2. Generic task state machine used at design time [11]

Developers may extend each transition at design time, e.g., to denote that completing a task logout triggers the abortion of a task check out. In this example, the transition from running to completed of the logout task state machine may be extended by the generation of an Abort event that is sent to check out and its task state machine, respectively. Further actions may be added to transitions, e.g., to specify modifications of values of domain objects or to alter the Boolean values of constraints [11]. In addition to these effects resulting from task state changes the intended effect of a regular task execution is described by a so-called Behavior. 


\subsection{Behaviors and Conditions}

Behaviors specify the effects of task execution, i.e. the intended actions affecting the domain. They can be assigned only to elementary tasks (performed while the task is in the state running). Thus, a composite task doesn't possess any behavior. Its effect is given by the combination of its subtasks, which themselves may be elementary or composite tasks.

The meta-model contains different specializations of the concept Behavior, e.g. ObjectManipulationBehavior by which manipulations of domain objects are specified. Developers may extend the set of behaviors as needed. All in all, our way of connecting specific manipulations to task execution is similar to the use of userAction and taskItem (object of an action) described in [13, page 62]. Currently we also follow an object-oriented approach, thus an ObjectManipulationBehavior contains a field referencing an object's method. In the case the behavior is assigned to a task, the method is invoked if the task is performed. A further example of a behavior type is the RequestTaskStateChangeBehavior by which impacts on other tasks performance may be specified. Such a behavior is used in the example below that is given by showing the XML description internally used. If the AbortCheckOut behavior is executed an Abort event is sent to the task with the identification $46 \ldots$ (line 3 to 5).

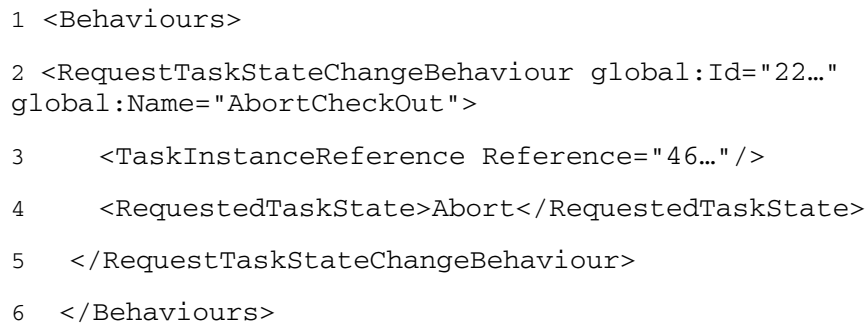

Conditions constrain task performance, e.g. a task can be started only if its preconditions hold true, while it can be finalized only if its post-conditions are fulfilled. The concept Condition enables to cope with such constraints. Similarly to behaviors predefined types exist, for example, an ObjectValueCondition is true if an attribute of a domain object holds a given value, otherwise the condition is false. By means of a TaskHasStateCondition it is evaluated whether or not a task is in a given state. In the example below checkout is performed is a TaskHasStateCondition. It is true if the task with the ID $4 D \ldots$ is in its Running state.

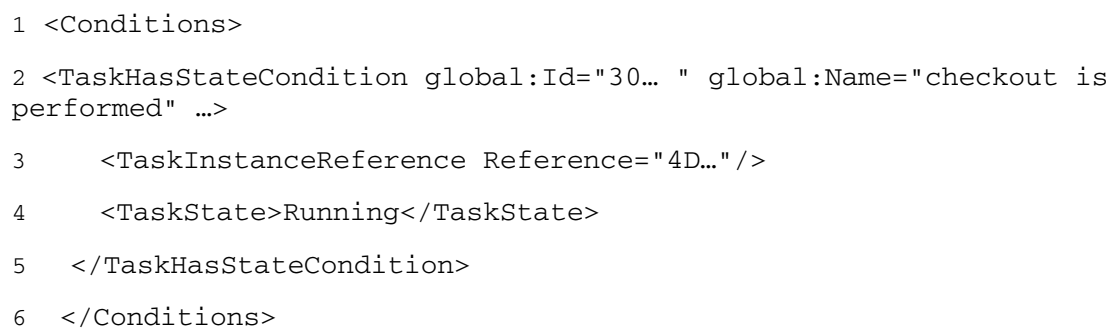


The following description exemplifies how behaviors and conditions are assigned to tasks by means of references. The condition checkout is performed is assigned as a pre-condition to the task empty shopping cart (line 2 to 4 ). The effect of this task is given by the behavior AbortCheckOut (line 6). Generally, usage of a condition as a pre- or post-condition depends on whether it is referenced in the PreConditions or in the PostConditions section.

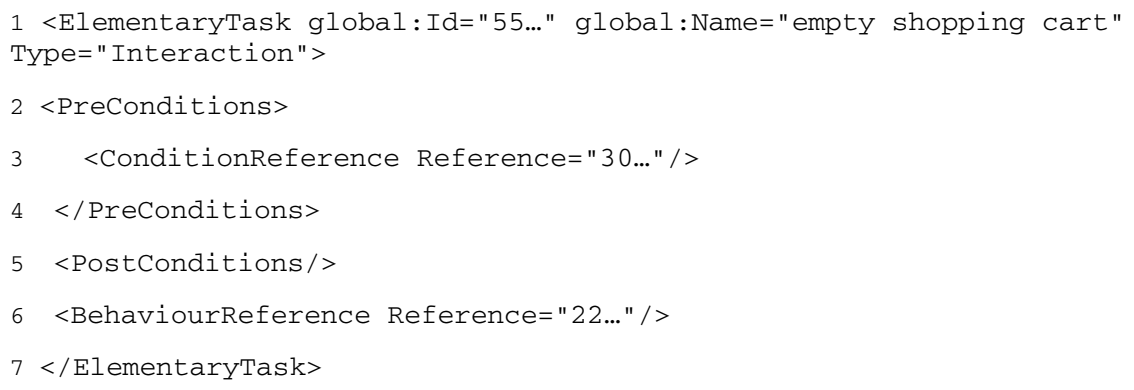

\subsection{Temporal Relationships}

A TemporalRelationship characterizes task sequencing and hereby the initial use of a set of modalities over time. A set of predefined relationships exists, such as Sequence (sequential task execution), Parallel (timely overlapping of task execution), Loop (repeated task execution). Further patterns, however, can be specified and added to the set. Temporal relationships are used within so-called temporal relationship structures to model the order of subtask execution.

A temporal relationship structure is described by means of a hierarchy within which a temporal relationship may include further ones, i.e. temporal relationships, similar to tasks, are structured hierarchically. Inner nodes represent tasks or further temporal relationships, but a leaf node must be a task. Hereby complex temporal dependencies can be specified - without introducing additional tasks only for the purpose of defining temporal ordering as it is required by, e.g., MAD [5] and in our former approach WTM [11].

In a multimodal interface for example, the user may gaze at a text document icon while repeatedly moving a picture causing the picture to be inserted at the beginning of the text document. In this example (see Fig. 3 (a)) the task insert picture exists that is composed of the elementary subtasks gaze at icon (possessing the ID 71...) and move picture (ID $5 E \ldots$... Subtasks are listed within the CompositeTask TAG (line 3 and 4) while their execution order is given by means of the TemporalRelationships Tag (line 6 to 13).

If the user, however, holds the picture over the icon for more than two seconds the document should be opened. Now the user may specify an exact position by moving the picture over the opened document and dropping it once the desired position is reached. Fig. 3 right hand shows the corresponding temporal structure. This time a tree notation depicting the temporal hierarchy instead of XML specification is used. It shows in terms of the example how a hierarchical temporal relationship structure is composed of subtasks and temporal relationships (rectangles with dotted lines). 
Generally, to each composite task a temporal relation structure is assigned. The occurrence of each subtask within the tree is restricted to exactly one: The temporal relation structure is defined for all subtasks the superior task is composed of, but each subtask can be referenced only one time.

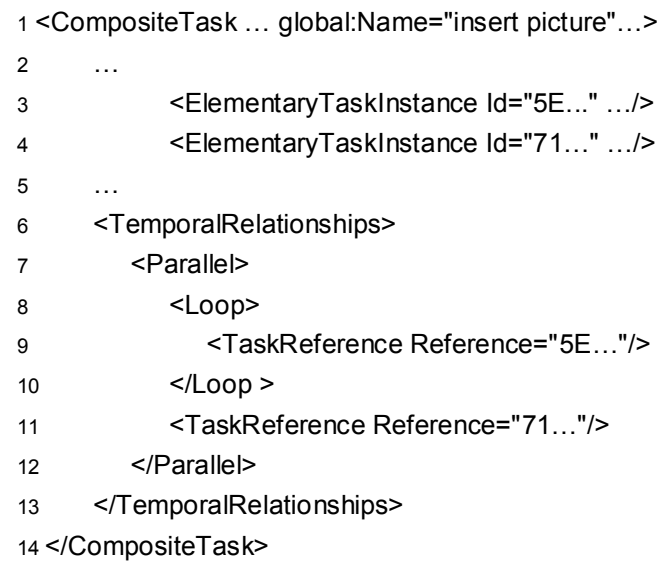

(a) a temporal structure given by means of XML specification

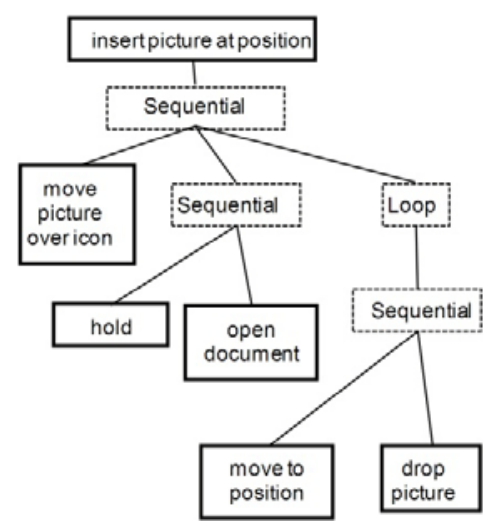

(b) a temporal structure visualized by hierarchical tree notation

Fig. 3. Temporal structures of inserting a picture

\section{Executable Task Model}

The executable task model is part of a runtime environment that is configured based on the static model specified at design time. The environment contains a TaskManager, a DomainManager, a BehaviorManager, a ConditionManager, and a TemporalRelationManager that interact which each other to realize the behavior as given by the static model. The managers responsible for behaviors, conditions and temporal relations are extended with the implementation of specific behaviors (such as ObjectManipulationBehavior and RequestTaskStateChangeBehavior), conditions (e.g. ObjectValueCondition and TaskHasStateCondition) and temporal relations (Sequence, Parallel, Loop, etc.), respectively. Adding, for example, a further temporal relation to our modeling approach requires two steps: First of all, the meta-model, i.e. its XML schema has to be extended with an according Tag so that the additional temporal relation can be used at design time. Secondly, the relation must be coded and added to the runtime environment.

\subsection{Generic Task Life Cycle at Runtime}

The kernel concept of the executable task model is a generic task lifecycle to handle and control task performance. It means that the approach presented in this paper makes use of a generic task lifecycle not only at design time (design task state machine, in short d-TSM) but also at runtime (referred to as r-TSM). 


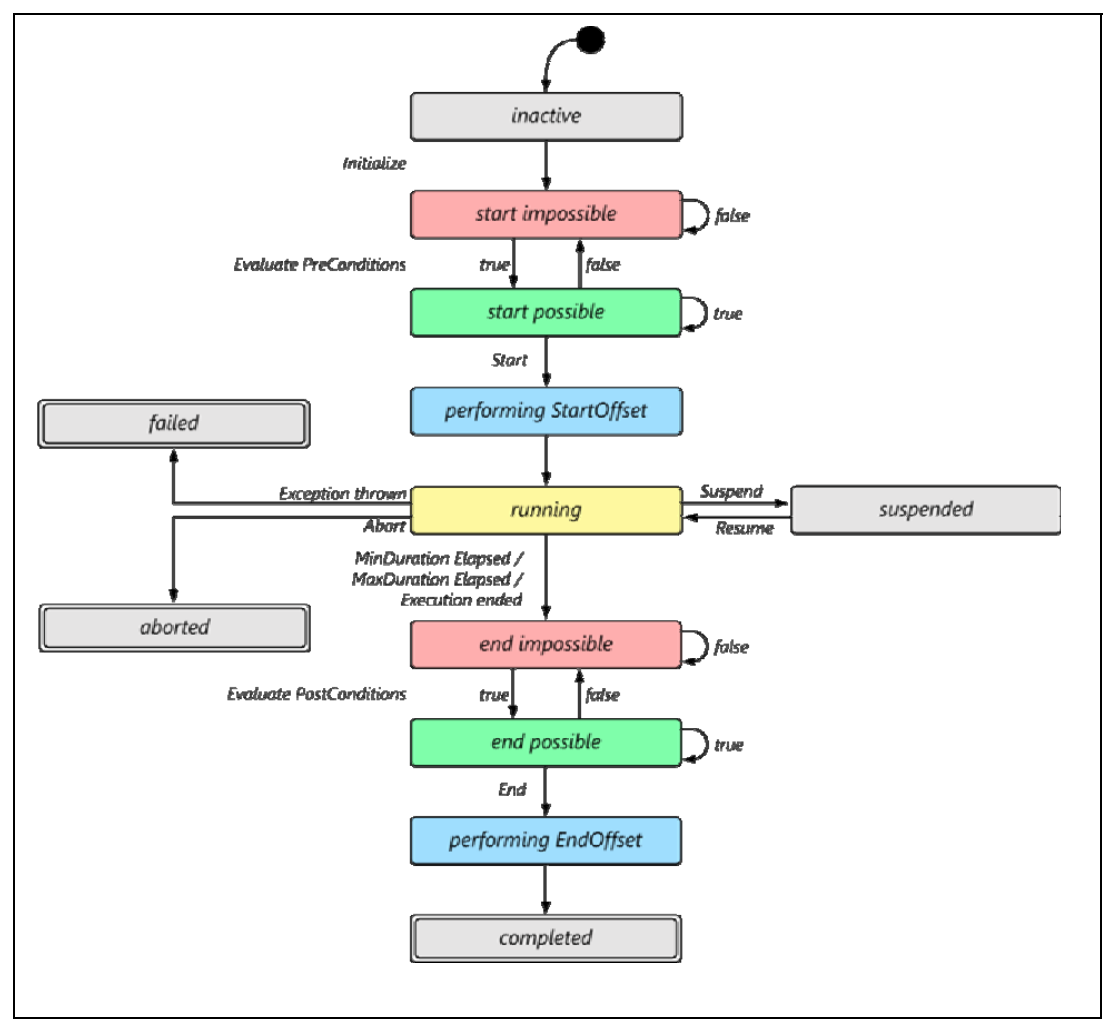

Fig. 4. Generic task state machine used at runtime

The generic task state machine of the runtime environment (see Fig. 4) is an extended version of the one introduced in Fig. 2 since additional runtime control information is required to handle task execution. All in all, the d-TSM describes the task life cycle from the user's point of view while the r-TSM describes it from the system's point of view. (Please note that the skip possibility is handled by the TemporalRelationManager and thus not modeled explicitly in the runtime task state machine.) The meanings of the states are as follows:

inactive: The task is part of the task space but not "accessible", i.e. the software object representing the task exists but is not initialized.

start impossible: Beginning the task is not possible because at least one of its preconditions is not fulfilled. Conditions are checked permanently. In the case a condition changed its Boolean value the task manager is informed, so that for all transitions depending on that condition it is evaluated whether they are to be triggered. A task remains in the state start impossible as long as at least one precondition is unfulfilled (false) and the state start possible is adopted as soon as all pre-conditions are true.

start possible: All pre-conditions of the task are fulfilled. The task state is set to start impossible again once one of the pre-conditions evaluates to false. However, if the 
Start event is received the task begins to be performed. This event may result from a user action and thus be generated by the UI.

performing StartOffset: The task is started and a defined time interval is waited before

it actually runs. As soon as the StartOffset elapses the state running is adopted. If no StartOffset is defined (i.e. its value is zero), the state running immediately becomes the current state.

running: This state denotes the actual performance of a task and it's subtasks, if applicable.

suspended: The task is interrupted because of the application logic, i.e. the events Suspend and Resume are triggered by the application.

end impossible: The task run cannot be ended because at least one of its postconditions is not fulfilled.

end possible: All post-conditions of the task are fulfilled. However, ending the task may depend on further dependencies. Therefore, only the End event denotes the end of task execution. Again, the event may result from UI interactions.

performing EndOffset: The task is ended and a defined time interval is waited before

it is completed. If the value of the EndOffset is zero, end possible immediately

becomes the current state.

completed: This state marks a successful task execution.

aborted: This state indicates an abort that is triggered by the application logic.

failed: The task performance fails because an exception is thrown.

\subsection{Configuration}

A state machine exists for each task in both the design model and the executable task model. Extensions of a d-TSM are transferred systematically to the r-TSM within the configuration process. Each transition extension of a task's generic d-TSM is added to the respective transition of its generic r-TSM. Hence, if the TaskManager performs a state transition all actions assigned to it are invoked. For example, in the case a transition is extended with a RequestTaskStateChangeBehavior a state-change event according to the given state in the behavior specification is sent to the task referenced in the behavior specification.

Conditions are introduced into r-TSM according to their specified usage in the design model: Pre-conditions of a task are assigned to its transitions between start impossible and start possible as (additional) requirements for performing these transitions. Post-conditions, in the same way, extend the transitions between end impossible and end possible.

StartOffset and EndOffset are represented by means of the states performing StartOffset and performing EndOffset, respectively. As described by the generic rTSM, the specified time has to elapse before the subsequent state is adopted. Fulfillment of durations constraining task execution (MinDuration and MaxDuration) is not realized by an explicit state but controlled by the TaskManager while the task is in running. Additionally, in the case of a composite task the r-TSM of its subtasks are considered by the execution process according to the defined temporal structure.

Temporal relations are systematically transferred to the executable model as well. Here the hierarchies of both the tasks and the temporal structures are regarded. Thus, temporal relations are fulfilled in close cooperation between the TaskManager and the 
TemporalRalationManager. A composite task, as mentioned above, possesses a list of all its subtasks and a hierarchical temporal structure. The last one is composed of temporal relations (inner nodes) and task references (leaf nodes). Loading the XML specification of a temporal structure results first of all in the internal representation of its hierarchy. Fig. 5 (a) shows the temporal hierarchy resulting from the example depicted in Fig. 3 (a). The TemporalRalationManager instantiates for each relation an according control object. However, this is only possible if the runtime environment contains an extension implementing the relation's semantics. In the case of a temporal relation Tag without corresponding implementation a warning is generated and the relation is ignored by substituting it with the initial NoOrder relation. The task references given in the model specification remain but are replaced at runtime by the software objects representing the referenced tasks (see Fig. 5 (b)). Furthermore, the software objects realizing behaviors are analogously linked to tasks. Fig. 6 (b) shows the order of task execution for the simple example insert picture, in which the behaviors of move picture and of gaze at icon are given by behaviorMP and behaviorGI, respectively.

(a)

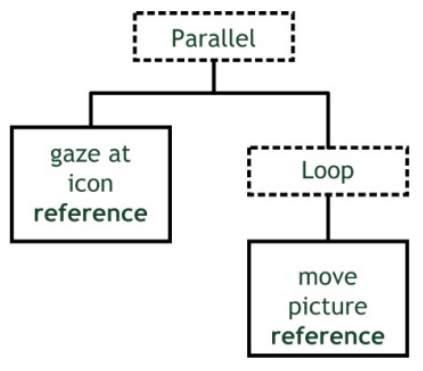

(b)

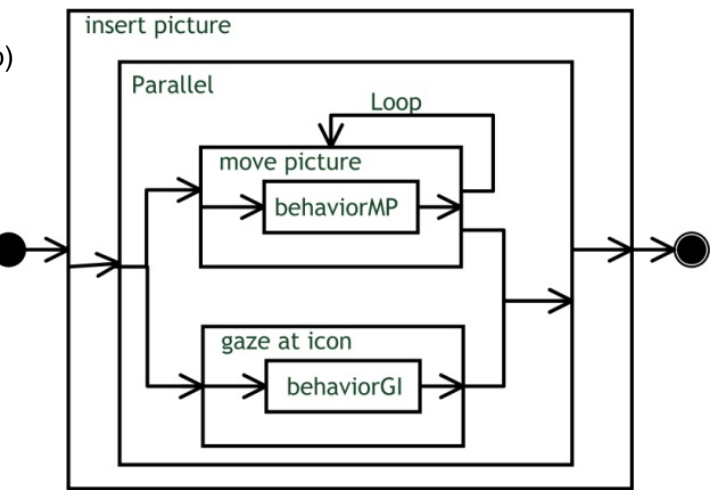

Fig. 5. Temporal Structure after (a) Loading and (b) at Runtime

\section{State of Work}

The meta-model is formally described by means of XML Schema. First versions of a task editor, a runtime system executing the models and a simulator based on it are developed. Both the editor and the simulation tool are in the state of proof of concept tools. The focus was on extensibility and on temporal dependencies. Easy extensibility was reached by separating the concerns of the concepts Task, Condition, Temporal Relation, Behavior and Domain Object that are linked to each other by means of references, processed by dedicated runtime managers. The focus on temporal dependencies is motivated by multimodal user interfaces, in which complex relations may exists. At task layer we abstract from specific usage of interaction modalities as far as possible and describe the impact of their use on task performance over time. Task frames define the time intervals that are structured by means of hierarchical ordering of temporal relationships and sub-tasks. The editor and the simulation tool mentioned above make use of nearly the same diagrams. The representations and view concepts, 
however, are to be investigated in follow-up work. A step in this direction is our current work on improving the task state machine editor.

Acknowledgments. This work was supported by the German Federal Ministry of Education and Research.

\section{References}

1. Calvary, G., Coutaz, J., Thevenin, D., Limbourg, Q., Bouillon, L., Vanderdonckt, J.A.: Unifying Reference Framework for Multi-Target User Interfaces. Interacting with Computers 15(3), 289-308 (2003)

2. Bomsdorf, B., Szwillus, G.: Early Prototyping based on Executable Task Models. In: Human Factors in Computing Systems, Conference Companion CHI 1996, pp. 254-255 (1996)

3. Mori, G., Paterno, F., Santoro, C.: CTTE: support for developing and analyzing task models for interactive system design. IEEE Transactions on Software Engineering 28(8), 797-813 (2002)

4. Uhr, H.: TOMBOLA: Simulation and User-Specific Presentation of Executable Task Models. In: Proc. of the International HCI Conference, pp. 263-267 (2003)

5. Caffiau, S., Girard, P., Scapin, D., Guittet, L., Sanou, L.: Formally Expressing the Users' Objects World in Task Models. In: England, D., Palanque, P., Vanderdonckt, J., Wild, P.J. (eds.) TAMODIA 2009. LNCS, vol. 5963, pp. 117-130. Springer, Heidelberg (2010)

6. Klug, T., Kangasharju, J.: Executable Task Models. In: TAMODIA 2005 Proceedings of the 4th International Workshop on Task Models and Diagrams, pp. 119-122 (2005)

7. Betermieux, S., Bomsdorf, B.: Task-Driven Composition of Web User Interfaces. In: CADUI 2008, 7th International Conference on Computer-Aided Design of User Interfaces, pp. 233-244 (2008)

8. Clerckx, T., Vandervelpen, C., Coninx, K.: Task-based design and runtime support for multimodal user interface distribution. In: Gulliksen, J., Harning, M.B., van der Veer, G.C., Wesson, J. (eds.) EIS 2007. LNCS, vol. 4940, pp. 89-105. Springer, Heidelberg (2008)

9. Bouchet, J., Nigay, L., Ganille, T.: ICARE: a component-based approach for the design and development of multimodal interfaces. In: CHI Extended Abstracts 2004, pp. 13251328 (2004)

10. Blumendorf, M., Lehmann, G., Feuerstack, S., Albayrak, Ş.: Executable Models for Human-Computer Interaction, In Interactive Systems. In: Graham, T.C.N. (ed.) DSV-IS 2008. LNCS, vol. 5136, pp. 238-251. Springer, Heidelberg (2008)

11. Bomsdorf, B.: The WebTaskModel Approach to Web Process Modelling. In: Winckler, M., Johnson, H. (eds.) TAMODIA 2007. LNCS, vol. 4849, pp. 240-253. Springer, Heidelberg (2007)

12. Allen, J.F.: Maintaining Knowledge about Temporal Intervals. Communications of the ACM 26(11), 832-843 (1983)

13. Stanciulescu, A.: A Methodology for Developing Multimodal User Interfaces of Information System, Ph.D. thesis, Université catholique de Louvain, Louvain-la-Neuve, Belgium (2008) 